\title{
ON THE BEHAVIOR OF INTEGRAL FUNCTIONS IN DISTANT PORTIONS OF THE PLANE*
}

\author{
BY W. B. FORD
}

1. Introduction. In a series of remarkable papers extending over the years 1904-08 Mr. E. W. Barnes $\dagger$ has determined the asymptotic behavior in the neighborhood of the point at infinity of various types of analytic functions. The types considered are distinguished from each other by the forms of their Maclaurin developments, these being regarded as given and forming the starting point of the investigation in each case. $\ddagger$ In point of method, Barnes makes extensive use of contour integrations and the calculus of residues. In point of results, his emphasis falls rather upon the detailed study of each individual function-type considered than upon the attainment of one or more general theorems which may be regarded as central in character to the subject as a whole. In my book on divergent series (see footnote below) I have already indicated on page 60 a general theorem through which the asymptotic character of a wide variety of special functions, including some of those considered by Barnes, may be readily determined $\S$ and I desire in the present paper to indicate another such theorem, this being of a nature supplementary to the former one. It may be applied

* Presented to the Society, September 8, 1927.

$\dagger$ Now the Bishop of Birmingham (England).

$\ddagger$ A list of the papers referred to may be found in my book entitled Studies on Divergent Series and Summability (Michigan Science Series, vol. II, 1916) page 184 . The various function-types considered by Barnes are summarized in his paper entitled The asymptotic expansion of integral functions defined by Taylor's series appearing in the Philosophical Transactions of the Royal Society of London, vol. 206 (1906), pp. 249-297.

$\$$ I take this occasion to note that the relation $w=0,1,2,3, \cdots$, occurring in line 6 of the theorem should be corrected to read $w=0, \pm 1, \pm 2, \pm 3, \cdots$. 
in particular to the special function designated by Barnes as $G_{\beta}(z, \theta)$ and defined as follows:

$$
G_{\beta}(z, \theta)=\sum_{n=0}^{\infty} \frac{z^{n}}{\Gamma(n+1)(n+\theta)^{\beta}},
$$

where $\beta$ and $\theta$ are any constants, real or complex, except that $\theta \neq 0,-1,-2, \cdots$. In $\$ 4$ I shall show, in fact, how the theorem may be applied to this special function.

As to method, I find that the elementary theory of differential equations suffices to establish one of the two parts of the theorem. For the other part, I employ the calculus of residues in the manner common to such investigations.

Wherever the symbol $R(z)$ occurs it is to be understood as meaning the real part of $z$.

2. TheOREM.

Part I. $R(z)>0$. If, when considered for large (positive integral) values of $n$, the function $g(n)$ occurring in the coefficient of the power series

$$
\sum_{n=0}^{\infty} \frac{g(n)}{\Gamma(n+k)} z^{n} ; k=\text { any given constant (real or complex) }
$$

may be developed in either a convergent or asymptotic series of the form

$$
\begin{aligned}
g(n)=c_{0} & +\frac{c_{1}}{n+k}+\frac{c_{2}}{(n+k)(n+k+1)}+\cdots \\
& +\frac{c_{p}+\delta(p, n)}{(n+k)(n+k+1) \cdots(n+k+p-1)}
\end{aligned}
$$

where $c_{0}, c_{1}, c_{2}, \cdots$ are constants and $\lim _{n \rightarrow \infty} \hat{\delta}(p, n)=0$, then the function $f(z)$ defined by the series (2), when considered for values of $z$ for which $R(z)>0$, may be developed asymptotically in the form

$$
f(z) \sim e^{z} z^{1-k}\left[c_{0}+\frac{c_{1}}{z}+\frac{c_{2}}{z^{2}}+\frac{c_{3}}{z^{3}}+\cdots\right] .
$$


Part II. $R(z)<0$. If the function $g(n)$ may be regarded as a function $g(w)$ of the complex variable $w=x+i y$ and as such satisfies the following two conditions: (a) it is single valued and analytic throughout the finite w-plane and (b) it remains less than a constant when considered for all values of $w$ of large modulus and of real part greater than any arbitrary fixed value (positive or negative), then the function $f(z)$ defined by (2), when considered for values of $z$ for which $R(z)<0$, may be developed asymptotically in the form

$$
\begin{aligned}
f(z) \sim-\frac{g(-1)}{\Gamma(k-1)} & \frac{1}{z}-\frac{g(-2)}{\Gamma(k-2)} \frac{1}{z^{2}} \\
& -\frac{g(-3)}{\Gamma(k-3)} \frac{1}{z^{3}}-\cdots .
\end{aligned}
$$

It is to be observed at once that under the conditions imposed in either part I or part II of the theorem, the series (2) necessarily has an infinite radius of convergence, thus rendering $f(z)$ a so-called integral function. In this particular the theorem is in contrast to the former one referred to in $\$ 1$.

In order to prove the theorem, let us begin by considering the special function defined by (2) in case $g(n)=1$; that is, the function which we shall call $F(z)$, defined as follows:

$$
F(z)=\sum_{n=0}^{\infty} \frac{z^{n}}{\Gamma(n+k)} .
$$

Upon multiplying both sides of this relation by $z^{k-1}$ and subsequently taking the derivative of each member of the result, making use of the relation

$$
\Gamma(n+k)=(n+k-1) \Gamma(n+k-1),
$$

we find that

$$
\begin{aligned}
\frac{d}{d z}\left[z^{k-1} F(z)\right] & =\frac{z^{k-2}}{\Gamma(k-1)}+z^{k-1} \sum_{n=0}^{\infty} \frac{z^{n}}{\Gamma(n+k)} \\
& =\frac{z^{k-2}}{\Gamma(k-1)}+z^{k-1} F(z) .
\end{aligned}
$$


Thus it appears directly that $F(z)$ satisfies the differential equation

$$
F^{\prime}(z)+\frac{k-1-z}{z} F(z)=\frac{1}{z \Gamma(k-1)} .
$$

This equation, being linear and of the first order, is readily solved by elementary methods giving as its general solution

$$
F(z)=\frac{e^{z} z^{1-k}}{\Gamma(k-1)} \int_{c}^{z} e^{-z} z^{k-2} d z,
$$

where $c$ is an arbitrary constant. In order that this $F(z)$ shall coincide with the particular $F(z)$ defined by (6) we need only to determine $c$ in such a manner that the limit approached by (7) as $z$ approaches zero shall be the first term of (2); that is, shall be $1 / \Gamma(k)$. Moreover, this will be the case if we take $c=0$, it being assumed for the moment (in order that the integral involved may assuredly have a meaning) that $R(k)>1$. In fact, with $c=0$ in (7) we have as desired

$$
\begin{aligned}
\lim _{z \rightarrow 0} F(z) & =\frac{1}{\Gamma(k-1)} \lim _{z \rightarrow 0} \frac{\int_{0}^{z} e^{-z} z^{k-2} d z}{z^{k-1}}=\frac{0}{0} \\
& =\left[\frac{e^{-z} z^{k-2}}{\Gamma(k-1)(k-1) z^{k-2}}\right]_{z=0}=\frac{1}{\Gamma(k)} .
\end{aligned}
$$

Whenever $R(k)>1$ we may therefore express the given $F(z)$ of (6) as follows:

$$
F(z)=\frac{e^{z} z^{1-k}}{\Gamma(k-1)} \int_{0}^{z} e^{-z} z^{k-2} d z .
$$

If we now confine ourselves to values of $z$ for which $R(z)>0$, we may write (8) in the form

$$
F(z)=\frac{e^{z} z^{1-k}}{\Gamma(k-1)}\left[\int_{0}^{\infty} e^{-z} z^{k-2} d z-\int_{z}^{\infty} e^{-z} z^{k-2} d z\right],
$$

where it is understood that the integration in the first integral takes place along the positive real axis from $z=0$ to $z=+\infty$, 
while in the second integral it takes place in the direction of the positive real axis from $z=z$ to $z=\infty$.

We have now but to recall that

$$
\int_{0}^{\infty} e^{-z} z^{k-2} d z=\Gamma(k-1)
$$

in order to have

(9) $\quad F(z)=e^{z} z^{1-k}\left[1-\frac{1}{\Gamma(k-1)} \int_{z}^{\infty} e^{-z} z^{k-2} d z\right] ; R(z)>0$.

The expression here appearing is seen to have a meaning not only under the tentative assumption $R(k)>1$, which was introduced above, but for all values (real or complex) of $k$. In fact, it is analytic throughout the finite $k$-complex plane, and, inasmuch as the same is true of the expression occurring on the right in (6), it follows that, whatever the value of $k$, $F(z)$ is given by (9) so long as $R(z)>0$. Moreover, with $R(z)$ thus limited, we see that as mod $z$ increases indefinitely the integral appearing in (9) becomes an infinitesimal of order higher than $(\bmod z)^{-m}$, where $m$ is any assigned positive integer. Hence, if $R(z)>0$, we may write

$$
F(z)=e^{z} z^{1-k}[1+\eta(z)] ; \quad \lim _{|z| \rightarrow \infty} z^{m} \eta(z)=0,
$$

where $m=0,1,2,3, \cdots$. To state this in the language of the theory of asymptotic series, we thus may write when $R(z)>0$,

or simply

$$
F(z) \sim e^{z} z^{1-k}\left[1+\frac{0}{z}+\frac{0}{z^{2}}+\frac{0}{z^{3}}+\cdots\right],
$$

$$
F(z) \sim e^{z} z^{1-k} .
$$

This preliminary result having been established, we proceed to establish part I of the theorem. In order to avoid unnecessary repetition of statement, let it be henceforth understood until the contrary is stated that the only values of $z$ under consideration are those for which $R(z)>0$. 
Having chosen an arbitrarily large positive integer $m$, we may write the function $f(z)$ defined by $(2)$ in the form

$$
f(z)=\phi(z, m)+\psi(z, m),
$$

where

$$
\phi(z, m)=\sum_{n=0}^{m-1} \frac{g(n)}{\Gamma(n+k)} z^{n}, \quad \psi(z, m)=z^{m} \sum_{n=0}^{\infty} \frac{g(m+n)}{\Gamma(n+k+m)} z^{n} .
$$

By choosing $m$ sufficiently large, the coefficient $g(m+n)$ may be developed for all values of $n$ in the form (3). If this be done, $\psi(z, m)$ takes the following form:

where

$$
\begin{aligned}
\psi(z, m)= & c_{0} z^{m} \sum_{n=0}^{\infty} \frac{z^{n}}{\Gamma(n+k+m)} \\
& +z^{m} \sum_{r=1}^{p} c_{r} \sum_{n=0}^{\infty} \frac{z^{n}}{\Gamma(n+k+m+r)}+R(z, m, p),
\end{aligned}
$$

$$
R(z, m, p)=z^{m} \sum_{n=0}^{\infty} \frac{\delta(m+n, p)}{\Gamma(n+k+m+p)} .
$$

Considering now the first two expressions appearing on the right in (13), if we apply (11) to the first of these, using the appropriate value of $k$, namely, $k=k+m$, we see that for values of $z$ of large modulus it becomes asymptotic to $c_{0} z^{m} e^{z} z^{1-k-m}=c_{0} e^{z} z^{1-k}$. Similarly, the second of the expressions in question consists of $p$ series of which the $r$ th is seen to be asymptotic to $c_{r} e^{z} z^{1-k-r}$, when $\bmod z$ is large. Hence, $\psi(z, m)$ is of the form

$$
\psi(z, m)=\Omega(z, m, p)+R(z, m, p)
$$

where

$$
\Omega(z, m, p) \sim e^{z} z^{1-k}\left[c_{0}+\frac{c_{1}}{z}+\frac{c_{2}}{z^{2}}+\cdots+\frac{c_{p}}{z^{p}}\right] .
$$

We shall now show that when $\bmod z$ is large $\psi(z, m)$ may be developed asymptotically in the analogous series (instead of polynomial) form as follows: 


$$
\psi(z, m) \sim e^{z} z^{1-k}\left[c_{0}+\frac{c_{1}}{z}+\frac{c_{2}}{z^{2}}+\cdots\right] .
$$

In order to do this, it suffices in view of (15) and (16) to show that, having chosen an arbitrarily small positive quantity $\epsilon$, we may determine $m$ such that, by subsequently taking $\rho=\bmod z$ sufficiently large, we may write

$$
|R(z, m, p)|<\frac{K \epsilon e^{\rho} \rho^{1-k}}{\rho^{p}}=K \epsilon e^{\rho} \rho^{1-p-k} ; K=\text { const. }
$$

This relation, in fact, may be brought about as follows: For the given $\epsilon$ choose $m$ so large that $|\delta(m, p)|<\epsilon$. Then we shall likewise have necessarily $|\delta(m+n, p)|<\epsilon ; n=0,1,2$, $3, \cdots$. From the definition (14) of $R(z, m, p)$, we may then write

$$
|R(z, m, p)|<\rho^{m} \epsilon \sum_{n=0}^{\infty} \frac{\rho^{n}}{\Gamma(n+k+m+p)} .
$$

Applying (10) with $k+m+p$ used for $k$, we thus have

$$
\begin{aligned}
|R(z, m, p)| & <\rho^{m} \epsilon e^{\rho} \rho^{1-(k+m+p)}[1+\eta(m, p, z)] \\
& =\epsilon e^{\rho} \rho^{1-p-k}[1+\eta(m, p, z)],
\end{aligned}
$$

where $\lim _{|z| \rightarrow \infty} \eta(m, p, z)=0$. This result at once yields the desired relation (18), thus establishing (17).

We have now but to observe that $\phi(z, m)$, being a polynomial in $z$, is such that

$$
\phi(z, m) \sim e^{z} z^{1-k}\left[0+\frac{0}{z}+\frac{0}{z^{2}}+\frac{0}{z^{3}}+\cdots\right],
$$

in order to obtain from (12), (17) and (19) the desired result (4) of part I of the theorem.

We therefore proceed to the proof of part II of the theorem. For this we shall employ the following fundamental statement from the calculus of residues:*

* The statement may be regarded as an immediate corollary of the fundamental theorems of Cauchy in the theory of functions of a complex variable. Details of a proof may be found, however, on page 9 of my Divergent Series, etc. already mentioned. 
"If $P(w)$ and $Q(w)$ are any two functions of the complex variable $w$ both of which are single valued and analytic throughout a region $A$ of the w-plane and of which $Q(w)$ vanishes within $A$ only at the points $w=\lambda_{1}, \lambda_{2}, \lambda_{3}, \cdots, \lambda_{n}$ which are zeros of the first order, and if $C_{n}$ denotes any closed contour lying within $A$ and including the points $w=\lambda_{1}, \lambda_{2}, \lambda_{3}, \cdots, \lambda_{n}$, then we may write

$$
\frac{1}{2 \pi i} \int_{C_{n}} \frac{P(w)}{Q(w)} d w=\sum_{n=1}^{n} \frac{P\left(\lambda_{n}\right)}{Q^{\prime}\left(\lambda_{n}\right)},
$$

where the indicated integration is taken about the entire contour $C_{n}$ in the positive direction."

Formula (20) being premised, let us take for our present purposes

$$
P(w)=\frac{\pi g(w)(-z)^{w}}{\Gamma(w+k)}, \quad Q(w)=\sin \pi w,
$$

where $g(w)$ is the function described in part II of the theorem, $z$ being regarded for the present as having any fixed value. Moreover, for the contour $C_{n}$, let us take the rectangle in the w-plane formed by the straight lines

$$
w=a-\frac{1}{2}+i y, \quad w=\frac{1}{2}+2 n+i y, \quad w=x \pm i j,
$$

where $a$ is any arbitrarily large negative integer, $n$ is an arbitrarily large positive integer and $j$ is any arbitrarily large positive quantity. Applying (20), we then obtain the relation

$$
\begin{aligned}
\frac{1}{2 i} \int_{C_{n}} \frac{g(w)(-z)^{w} d w}{\Gamma(w+k) \sin \pi w}=\sum_{n=a}^{2 n} \frac{g(n) z^{n}}{\Gamma(n+k)} \\
=\sum_{n=a}^{-1} \frac{g(n) z^{n}}{\Gamma(n+k)}+\sum_{n=0}^{2 n} \frac{g(n) z^{n}}{\Gamma(n+k)},
\end{aligned}
$$

the last summation on the right thus becoming the sum of the first $2 n$ terms of the given series (2). As to the integral on the left, we shall assume that the function $(-z)^{w}$ appearing in the integrand is rendered precise in meaning for any given 
value of $z=\rho(\cos \phi+i \sin \phi)$ through the following convention:

$$
(-z)^{w}=e^{w \log (-z)}=e^{w[\log \rho+i(\phi+\pi)]} ;-2 \pi<\phi \leqq 0 .
$$

Supposing at first that $z$ is real and negative; that is, that $\phi=-\pi$, we now proceed to study in detail the integral appearing in (21).

First, along the side of the rectangle upon which $w=x+i j$, we have $d w=d x$ and $\sin \pi w=\sinh \pi j(\sin \pi x \operatorname{ctnh} \pi j+i \cos \pi x)$, so that, if we designate by $I$ the contribution to the integral from this side, we may write

$$
I=\frac{(-z)^{i j}}{2 i \sinh \pi j} \int_{2 n+1 / 2}^{a-1 / 2} \frac{g(x+i j)(-z)^{x} d x}{\Gamma(x+k+i j)(\sin \pi x \operatorname{ctnh} \pi j+i \cos \pi x)} .
$$

Now, as $j$ is allowed to increase indefinitely, $\sinh \pi j$ becomes infinite like $e^{\pi j}$, while $|\Gamma(x+k+i j)|$ vanishes to as high an order as that of $e^{-\pi j / 2} ;^{*}$ also we have $\lim _{j \rightarrow \infty}$ ctnh $\pi j=1$. Hence, recalling the condition (b) of part II of the theorem, we see that $\lim _{j \rightarrow \infty} I=0$.

Similarly, the contribution to the contour integral in (21) arising from the side of $C_{n}$ upon which $w=x-i j$, is seen to be the same as (23), except that $-j$ occurs throughout instead of $j$. We have then but to recall that as $j$ increases indefinitely $\sinh (-\pi j)$ becomes infinite like $e^{\pi j}$ while $|\Gamma(x+k-i j)|$ vanishes to as high an order as that of $e^{-\pi j / 2}$, to see that this contribution also vanishes in the limit as $j \rightarrow \infty$.

Next, let us consider the contribution from the side of $C_{n}$ upon which $w=1 / 2+2 n+i y$. Here we have $d w=i d y$ and $\sin \pi w=\cos i \pi y=\cosh \pi y$, so that, having taken $j=\infty$ as indicated above, the contribution in question becomes

* See for example formula (10), page 24 of Nielsen's Handbuch der Theorie der Gammafunction. The formula there occurring, however, contains a typographical error inasmuch as the letter $u$ which appears under the radical should be $v$. 


$$
\begin{aligned}
J & =\frac{(-z)^{1 / 2+2 n}}{2} \int_{-\infty}^{\infty} \frac{g\left(2 n+\frac{1}{2}+i y\right)(-z)^{i y}}{\Gamma\left(2 n+k+\frac{1}{2}+i y\right) \cosh \pi y} d y \\
& =\frac{z^{1 / 2+2 n}}{2} \int_{-\infty}^{\infty} \frac{g\left(2 n+\frac{1}{2}+i y\right) e^{i y \log \rho}}{\Gamma\left(2 n+k+\frac{1}{2}+i y\right) \cosh \pi y} d y .
\end{aligned}
$$

That the improper integral here appearing has a meaning follows when we recall that as $y$ increases indefinitely in the positive sense, $\Gamma(2 n+k+1 / 2+i y)$ vanishes to as high an order as that of $e^{-\pi y / 2}$, and that as $y$ increases indefinitely in the negative sense, the same function vanishes to as high an order as that of $e^{\pi y / 2}$; also that, by virtue of (b), $g(2 n+1 / 2+i y)$ remains less than a constant whatever the value of $y$. Moreover, we may now show that $\lim _{n \rightarrow \infty} J=0$. In fact, this appears by writing $J$ in the form

$$
\begin{gathered}
J=\frac{g(2 n) z^{2 n}}{\Gamma(2 n+k)} \cdot \frac{z^{1 / 2}}{2} \int_{-\infty}^{\infty} \frac{g\left(2 n+\frac{1}{2}+i y\right)}{g(2 n)} \\
\cdot \frac{\Gamma(k+2 n)}{\Gamma\left(2 n+k+\frac{1}{2}+i y\right)} \cdot \frac{e^{i y \log \rho}}{\cosh \pi y} d y,
\end{gathered}
$$

and recalling that, inasmuch as the series (2) has an infinite radius of convergence, we have

$$
\lim _{n \rightarrow \infty} \frac{g(2 n) z^{2 n}}{\Gamma(2 n+k)}=0,
$$

whatever the value of $z$; also recalling our hypothesis (b) and the well known properties of the $\Gamma$ function.

If we now take account of the contribution to the integral in (21) arising from the remaining side $w=a-1 / 2+i y$ of $C_{n}$, noting that we here have $d w=i d y, \sin \pi w=(-1)^{a-1} \cosh \pi y$ while the integration takes place from $y=+\infty$ to $y=-\infty$, the fundamental relation (21) takes the following form ( $n$ having been allowed to increase indefinitely as indicated above):

$$
\begin{gathered}
\frac{(-1)^{a}(-z)^{a-1 / 2}}{2} \int_{-\infty}^{\infty} \frac{g\left(a-\frac{1}{2}+i y\right)(-z)^{i y}}{\Gamma\left(a+k-\frac{1}{2}+i y\right) \cosh \pi y} d y \\
=\sum_{n=a}^{0} \frac{g(n) z^{n}}{\Gamma(n+k)}+f(z) .
\end{gathered}
$$


Thus far we have confined $z$ to values which are real and negative. Suppose now that $z$ as appearing in (24) is allowed to take on complex values. The right side of (24) is evidently analytic for all such values of $z$. We shall now show that the left side is likewise an analytic function of $z$ so long as $R(z)<0$; that is, so long as $-3 \pi / 2<\phi<-\pi / 2$. To establish this it suffices to show that the improper integral in (24) converges uniformly for values of $z$ confined to such a region.* Now, the denominator of the integrand becomes infinite to as high an order as that of $e^{\pi y / 2}$ when $y \rightarrow+\infty$, and it becomes infinite to as high an order as that of $e^{-\pi y / 2}$ when $y \rightarrow-\infty$. As to the factor $(-z)^{i y}$, we have by (22)

$$
(-z)^{i y}=e^{i y[\log \rho+i(\phi+\pi)]}=e^{i y \log \rho} e^{-(\phi+\pi) y},
$$

and under the proposed restriction as regards $\phi$, that is, $-3 \pi / 2<\phi<-\pi / 2$, we have $-3 \pi / 2+\epsilon \leqq \phi \leqq-\pi / 2-\epsilon, \epsilon>0$, and hence $-\pi / 2+\epsilon \leqq \phi+\pi \leqq \pi / 2-\epsilon$. We now have but to recall again hypothesis (b) of part II of the theorem to see that, under the indicated restrictions as regards $\phi$, the integrand of (24) vanishes to as high an order as that of $e^{-\pi y / 2} e^{-(-\pi / 2+\epsilon) y}=e^{-\epsilon y}$ when $y \rightarrow+\infty$, and vanishes to as high an order as that of $e^{\pi y / 2} e^{-(\pi / 2-\epsilon) y}=e^{\epsilon y}$ when $y \rightarrow-\infty$. The uniform convergence property in question now follows immediately.

Inasmuch as (24) holds true, as we have now shown, for all negative values of $z$ and at the same time each side is an analytic function of $z$ so long as $R(z)<0$, it follows that it is likewise true for all such values of $z$. The proof of the theorem is thus complete.

3. Generalizations and Remarks. In order to secure simplicity of statement in the theorem the conditions (a) and (b) of part II were made unnecessarily restrictive. In fact, if one examines the proof of this part it appears readily that the following generalizations and remarks can be made.

* See for example Osgood, Encyklopädie der mathematischen Wissenschaften, II, $2, \S 6$. 
( $\alpha$ ) In condition (b) the statement "remains less than a constant" may be replaced by the following stronger statement: "is such that, to an arbitrarily small positive quantity $\eta$ there corresponds a positive constant $K$ (independent of $x$ and $y$ ) such that for all large values of $|x|$ and for all sufficiently large positive values of $y$ one may write

$$
\left|\frac{g(x \pm i y)}{g(x)}\right|<K e^{\eta y} . "
$$

$(\beta)$ In case condition (a) is not satisfied but instead the function $g(w)$, while still single valued throughout the finite $w$-plane, has $m(m \geqq 1)$ singularities situated at the points $w=w_{1}, w_{2}, w_{3}, \cdots, w_{m}$, then (5) continues to hold true provided one subtracts from the right member the sum of the residues of the function

$$
\frac{\pi g(w)(-z)^{w}}{\Gamma(w+k) \sin \pi w}
$$

at these points.

$(\gamma)$ In case condition (a) is not satisfied but instead the function $g(w)$, while still finite throughout the finite $w$-plane, is multiple valued owing to the presence of $p(p \geqq 1)$ branch points at $w=W_{1}, W_{2}, W_{3}, \cdots, W_{m}, \cdots, W_{p}$, then (5) continues to hold true provided that one adds to the right member the sum of the $p$ loop integrals of (25) of which the $m$ th is described as follows: Draw the straight line extending from the point $W_{m}$ to infinity in the direction of the positive (upper) half of the pure imaginary axis and let this line be regarded as a cut in the w-plane. Now let the loop consist of the two lines drawn on either side of and close to this cut, the ends of these lines near to the point $W_{m}$ being joined by a circular arc of small radius drawn about this point. Moreover, let the integration over the loop as thus formed be understood to be in the positive (counter-clockwise) direction.

In case the exceptions described in $(\beta)$ and $(\gamma)$ both occur in $g(w)$ at the same time, the alterations to be made in (5) will then consist of both those described above. 
Finally, it is to be noted that the theorem gives no information concerning the behavior of $f(z)$ in case $R(z)=0$. Naturally, this case calls for further investigation. We shall, however, omit it here, reserving it for possible discussion in a later paper.

4. Application to the Function $G_{\beta}(z, \theta)$. As stated in $\S 1$, we shall show in conclusion how the foregoing theorem, subject to the remarks in $\$ 3$, may be applied in particular to determine the asymptotic behavior of the function $G_{\beta}(z, \theta)$ studied by Barnes and defined by (1).

Wishing first to apply part I, we write (1) in the form

$$
G_{\beta}(z, \theta)=\sum_{n=0}^{\infty} \frac{g(n) z^{n}}{\Gamma(n+\beta+1)}
$$

where

$$
g(n)=\frac{\Gamma(n+\beta+1)}{\Gamma(n+1)(n+\theta)^{\beta}} .
$$

Now, it is a familiar fact immediately deducible from Stirling's formula for $\log \Gamma(x)$, that for values of $x$ whose real part is positive, we have

$$
\Gamma(x) \sim(2 \pi)^{1 / 2} x^{x-1 / 2} e^{-x}\left(1+\frac{a_{1}}{x}+\frac{a_{2}}{x^{2}}+\cdots\right),
$$

where $a_{1}, a_{2}, \cdots$ are constants. Hence

$$
\begin{gathered}
\Gamma(n+\beta+1) \sim(2 \pi)^{1 / 2} e^{-(n+\beta+1)}(n+\beta+1)^{n+\beta+1 / 2} \\
\cdot\left(1+\frac{a_{1}}{n+\beta+1}+\frac{a_{2}}{(n+\beta+1)^{2}}+\cdots\right) \\
\Gamma(n+1) \sim(2 \pi)^{1 / 2} e^{-(n+1)}(n+1)^{n+1 / 2}\left(1+\frac{a_{1}}{n+1}\right. \\
\left.+\frac{a_{2}}{(n+1)^{2}}+\cdots\right)
\end{gathered}
$$


and therefore

$$
\frac{\Gamma(n+\beta+1)}{\Gamma(n+1)} \sim e^{-\beta} \frac{(n+\beta+1)^{n+\beta+1 / 2}}{(n+1)^{n+1 / 2}}\left(1+\frac{b_{1}}{n}+\frac{b_{2}}{n^{2}}+\cdots\right),
$$

where $b_{1}, b_{2}, b_{3}$, etc. are constants whose values in terms of $a_{1}, a_{2}, a_{3}$, etc. may be readily determined. It follows that we may write

$$
\begin{aligned}
& \frac{\Gamma(n+\beta+1)}{\Gamma(n+1)(n+\theta)^{\beta}} \sim e^{-\beta}\left(1+\frac{\beta-\theta+1}{n+\theta}\right)^{\beta} \\
& \cdot\left(1+\frac{\beta}{n+1}\right)^{n+1 / 2}\left(1+\frac{b_{1}}{n}+\frac{b_{2}}{n^{2}}+\cdots\right) .
\end{aligned}
$$

The factor

$$
\left(1+\frac{\beta-\theta+1}{n+\theta}\right)^{\beta}
$$

here appearing is developable for large values of $n$ in a convergent series proceeding according to ascending powers of $1 / n$ while the factor

$$
\left(1+\frac{\beta}{n+1}\right)^{n+1 / 2}
$$

is developable for similar values of $n$ in a convergent series of the form

$$
e^{\beta}\left(1+\frac{c_{1}}{n}+\frac{c_{2}}{n^{2}}+\frac{c_{3}}{n^{3}}+\cdots\right) .
$$

It follows that we may write

$$
\frac{\Gamma(n+\beta+1)}{\Gamma(n+1)(n+\theta)^{\beta}} \sim 1+\frac{d_{1}}{n}+\frac{d_{2}}{n^{2}}+\frac{d_{3}}{n^{3}}+\cdots,
$$

where $d_{1}, d_{2}, d_{3}$, etc. are assignable constants. Hence, we may also write

$$
\begin{aligned}
& \frac{\Gamma(n+\beta+1)}{\Gamma(n+1)(n+\theta)^{\beta}} \sim 1+\frac{e_{1}}{n+\beta+1} \\
& \quad+\frac{e_{2}}{(n+\beta+1)(n+\beta+2)}+\cdots,
\end{aligned}
$$


where $e_{1}, e_{2}, e_{3}$, etc. are assignable constants. But (27) is the form (3) wherein $k=\beta+1$.

Applying (4), we therefore may write when $R(z)>0$

$$
G_{\beta}(z, \theta) \sim e^{z} z^{-\beta}\left(1+\frac{e_{1}}{z}+\frac{e_{2}}{z^{2}}+\frac{e_{3}}{z^{3}}+\cdots\right) .
$$

As to the values of the coefficients $e_{1}, e_{2}, e_{3}$, etc., they may evidently be computed to any desired point as follows, it being understood that $g(n)$ is defined by (26) and that the symbol $\Delta$ has its customary significance as used in the theory of finite differences; namely, $\Delta u(n)=u(n+1)-u(n)$ :

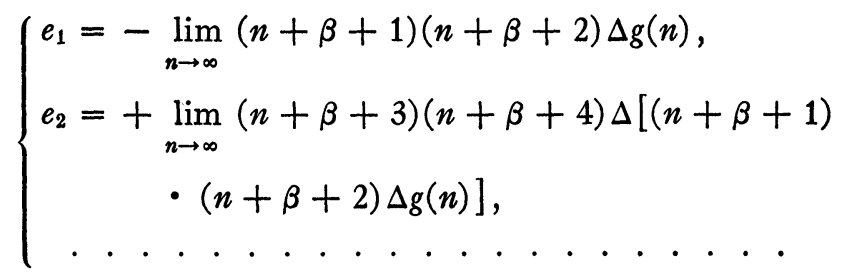

Moreover, these limits are not difficult to evaluate when one makes repeated use of the relation $\Gamma(n+1)=n \Gamma(n)$.

Wishing now to apply part II of the theorem to the present function $G_{\beta}(z, \theta)$, we note that we here have the series (2) in which $k=1$ and $g(n)=(n+\theta)^{-\beta}$. As the function $g(w)$ to be used in applying the theorem, we therefore naturally take

$$
g(w)=(w+\theta)^{-\beta} .
$$

This function at once satisfies condition (b) of the theorem so long as $\beta$ is a number having a positive, or zero, real part. In case $\beta$ has a negative real part the function

$$
\frac{g(x \pm i y)}{g(x)}=\left(\frac{x+\theta \pm i y}{x+\theta}\right)^{-\beta}=\left(1 \pm \frac{i y}{x+\theta}\right)^{-\beta}
$$

evidently satisfies the condition mentioned in $(\alpha)$ of $\$ 3$. In either case, therefore, the theorem may be applied so far as condition (b) is concerned. As regards condition (a), this will not be satisfied inasmuch as the function (30) will 
have a singular point in general at $w=-\theta$. However, to meet this situation we need only to apply either remark $(\beta)$ or $(\gamma)$ of $\S 3$ according as the singular point in question is a pole (in which case $\beta$ is a positive integer) or a branch point.

Applying (5), subject to the remarks $(\alpha),(\beta),(\gamma)$ of $\$ 3$, and noting also that inasmuch as $k=1$ in the present instance, all the coefficients of (5) are equal to zero, we conclude that when $R(z)<0$ we may write

$G_{\beta}(z, \theta) \sim L(z)+0+\frac{0}{z}+\frac{0}{z^{2}}+\cdots$, or simply $G_{\beta}(z, \theta) \sim L(z)$,

where $L(z)$ represents the negative of the residue of the function

$$
\frac{\pi(-z)^{w}}{\Gamma(w+1)(w+\theta)^{\beta} \sin \pi w}
$$

at the point $w=-\theta$ in case $\beta$ is an integer, or represents the loop integral of this function about the same point in the manner described in $(\gamma)$ of $\$ 3$ in case $\beta$ is not an integer.

Results (28) and (29) are essentially those arrived at by Barnes for the special function $G_{\beta}(z, \theta)$.* However, he obtains the coefficients $e_{1}, e_{2}, e_{3}$, etc. under a different form of definition from (29), and through an elaborate investigation he evaluates the loop integral referred to above as $L(z)$.

The University of Michigan

* See result (A), page 265, vol. 206A (1906) of the Philosophical Transactions of the Royal Society of London. 